\title{
O uso do programa de tratamento Stress Loading no tratamento da síndrome complexa de dor regional de tipo I
}

\section{The use of the Stress Loading treatment program in the treatment of complex regional syndrome of type I pain}

\author{
Thais Rosa Costa, Bárbara Santos Luccas Duarte, \\ Taísa Gomes Ferreira (In memorian), Aline Sarturi Ponte, \\ Miriam Cabrera Corvelo Delboni
}

Como citar este artigo: COSTA, THAIS R.: DUARTE, BÁRBARA S. L.; FERREIRA, TAÍSA G.; PONTE, ALINE S.; DELBONI, MIRIAM C. C.

O uso do programa de tratamento Stress Loading no tratamento da síndrome complexa de dor regional de tipo I. Revista Saúde (Sta. Maria). 2020; 46 (2)

\section{Autor correspondente:} Nome: Thais Rosa Costa E-mail: thaisrosacosta@gmail.com Telefone: (55) 99948-2852 Formação Profissional: Formada em Terapia Ocupacional pela Universidade Federal de Santa Maria (UFSM), Santa Maria, RS, Brasil.

Filiação Institucional: Universidade Federal de Santa Maria

Endereço para correspondência: Av. Roraima $n^{\circ} 1000$

Bairro: Camobi

Cidade: Santa Maria

Estado: Rio Grande do Sul

CEP: 97 105-900

Data de Submissão:

22/04/2020

Data de aceite:

01/08/2020

Conflito de Interesse: Não há conflito de interesse

(cc) BY-NC-ND

\section{RESUMO}

Este estudo o objetiva de analisar os benefícios da intervenção terapêutica ocupacional a partir da utilização do Programa de Tratamento Stress Loading, junto a duas pessoas acometidas pela Síndrome Complexa de Dor Regional de tipo I (SCDR I). Este caracteriza-se como um estudo de caso, descritivo, comparativo pré e pós tratamento. As intervenções ocorreram nos meses de maio a junho de 2014 e os instrumentos de coleta de dados utilizados foram Disabilities Arm Shoulder and Hand (DASH) e a Escala Visual Analógica (EVA). Participaram deste estudo duas mulheres, com 52 e 59 anos de idade, no momento do estudo ambas estavam afastadas de suas atividades laborais, mantinham acompanhamento fisioterapêutico e terapêutico ocupacional e utilizavam medicamentos antidepressivos e analgésicos. Pode-se observar que ambas as participantes apresentaram melhoras no escore do DASH e a EVA após as intervenções terapêuticas ocupacionais de seis semanas com o Programa de Tratamento Stress Loading.

PALAVRAS-CHAVE: Dor; Dor crônica; Dor intratável; Terapia Ocupacional.

\section{ABSTRACT}

This study aims to analyze the benefits of occupational therapeutic intervention from the use of the Stress Loading Treatment Program, with two people affected by Regional Pain Complex Syndrome Type I (RPCS I). This is characterized as a case study, descriptive, comparative pre and post treatment. The interventions took place from May to June 2014 and the data collection instruments used were Disabilities Arm Shoulder and Hand (DASH) and Visual Analog Scale (VAS). Two women, aged 52 and 59 , participated in this study. At the time of the study, both were away from their work activities, maintained physiotherapeutic and occupational therapeutic follow-up, and used antidepressants and analgesics. It can be observed that both participants presented improvements in the DASH score and VAS after the six-week occupational therapeutic interventions with the Stress Loading Treatment Program.

KEYWORDS: Pain; Chronic pain; Intractable pain; Occupational Therapy. 


\section{INTRODUÇÃO}

A dor é um sintoma que interfere de maneira significativa no cotidiano da pessoa, refletindo em seu bem-estar e na qualidade de vida. Sendo assim, deparam-se com prejuízos funcionais, psicológicos e sociais que podem resultar em comprometimentos na realização das suas principais ocupações relacionadas as áreas do autocuidado, produtividade e $\operatorname{lazer}^{1,2}$. O processo doloroso é um sintoma que acompanha quase todos os processos de deterioração da saúde, afeta diretamente a qualidade do sono, as interações sociais, as reações emocionais, a disposição e as habilidades físicas².

Em 1994 a Associação Internacional para o Estudo da Dor (IASP) ${ }^{3}$, classificou a dor como nociceptiva e neuropática. A dor nociceptiva é decorrente de uma ativação fisiológica de receptores ou da via dolorosa e a dor neuropática acionada por alguma lesão ou disfunção do sistema nervoso. A neuropática pode ser de origem central ou periférica que causam aos portadores, sofrimento e um alto nível de estresse.

Em 2011 o IASP revisou a classificação e adicionou mais um tipo, as dores disfuncionais ${ }^{4,5}$. Estas são doenças que tem em comum ausência de lesão tecidual clara que explique a síndrome dolorosa. $\mathrm{O}$ termo disfuncional refere-se ao funcionamento alterado do sistema nervoso, seja ele central ou periférico. Nesta classificação incluem-se a Neuralgia do Trigêmeo, a Fibromialgia e a Síndrome Complexa de Dor Regional de tipo I (SCDR I)6.

A SCDR I caracteriza-se como inconclusiva, com mecanismos biológicos pouco esclarecidos ${ }^{5}$, é um estado patológico complexo que envolve teorias inflamatórias e imunes, dor severa, edema, rigidez e alteração da cor do membro, resultante de um reflexo ou de uma resposta simpática anormal no organismo. As medidas terapêuticas realizadas junto as pessoas acometidas pela SCDR I buscam combater principalmente a dor exacerbada, o edema persistente e a rigidez, de forma a potencializar a funcionalidade aos indivíduos o mais brevemente possível, evitando rupturas drásticas em seu cotidiano.

Esta síndrome é mais comum em adultos e normalmente acomete um único membro, sendo o superior ou inferior?. A SCDR I não tem um código da Classificação Internacional de Doenças (CID) específico, mas pode ser reconhecida pelo CID R52.1, sendo classificada como dor crônica intratável. Segundo discussões realizadas nos estudos de Patterson e colaboradores ${ }^{5}$, a SCDR I é uma síndrome de difícil tratamento, a terapêutica aplicada pode dar-se de formas variadas. Dentre elas, existem o Programa de Tratamento Stress Loading recomendado por Watson, técnica essa utilizada no tratamento dos casos descritos esse artigo ${ }^{8}$.

Diante destas reflexões este estudo tem como objetivo analisar benefícios da intervenção terapêutica ocupacional a partir da utilização do Programa de Tratamento Stress Loading, junto a duas pessoas acometidas pela SCDR I. 


\section{MÉTODO}

Este estudo caracterizou-se como um estudo de caso, descritivo, comparativo pré e pós tratamento. Este desenvolveu-se vinculado ao Projeto de Pesquisa "Dor Neuropática nos Membros Superiores: Capacidades funcionais e aspectos biopsicossociais", este teve como objetivo identificar a capacidade funcional e os aspectos biopsicossociais que afetam o cotidiano destes sujeitos, para o aprimoramento das intervenções em Terapia Ocupacional. Ressalta-se que as ações do projeto tiveram início após a aprovação do Comitê de Ética em Pesquisa em Seres Humanos de uma Universidade pública do interior do Rio Grande do Sul, RS, sob o número do CAAE 08718312.4.0000.5346.

As intervenções ocorreram nos meses de maio a junho de 2014. Participaram deste estudo duas pessoas com diagnóstico médico de SCDR I encaminhados ao projeto pela Clínica de Dor de um Hospital Universitário do interior do Rio Grande do Sul. Ao convidar os mesmos para aderir ao projeto/intervenções foi esclarecido como iriam ocorrer as intervenções e solicitado a assinatura do Termo de Consentimento Livre e Esclarecido (TCLE), quando estes concordavam em participar.

O período de intervenções foi de seis semanas, organizados em encontros semanais, sendo que a primeira e última semanas foram destinadas para a avaliação e a reavaliação, respectivamente. Todas as intervenções realizadas neste estudo ocorreram no Ambulatório de Terapia Ocupacional de uma Universidade pública do interior do Rio Grande do Sul.

Para a avaliação e reavaliação foram utilizados os seguintes instrumentos: Disabilities Arm Shoulder and Hand (DASH) e a Escala Visual Analógica (EVA).

O DASH foi elaborado em inglês e adaptado para o idioma português por Santos e Gonçalvez em $2006^{9}$. Este instrumento é constituído por 30 questões, que envolvem componentes como: dor, fraqueza, rigidez, formigamento, AVD, além dos módulos opcionais para atletas e músicos, e outro para trabalhadores. As respostas às questões devem ser baseadas no desempenho ocupacional na semana anterior. 0 escore total varia de zero (0) a cem (100), onde zero (0) equivale à ausência de disfunção e cem (100) representa disfunção severa. O DASH é um instrumento que tem como objetivo mensurar os sintomas e função de sujeitos com patologias musculoesqueléticas nos membros superiores, sendo assim, a utilização deste por terapeutas ocupacionais pode auxiliar estes profissionais na identificação das as funções físicas, habilidades que estão mais comprometidas e sintomas que estão limitando o membro superior enquanto unidade funcional.

Para quantificar a intensidade do quadro álgico, utilizou-se a Escala Visual Analógica (EVA), escolheu-se este instrumento por ser de fácil compressão, reprodutível e sensível ao seu objetivo. Este é apresentado por Radomski ${ }^{10}$, como instrumento auto administrada que possibilita ao terapeuta ocupacional compreender a intensidade do quadro 
doloroso. A EVA é composta por uma linha com $10 \mathrm{~cm}$ de comprimento, numerada de zero (0) a dez (10), onde zero (0) - "sem nenhuma dor" e dez (10) representa o "pior nível de dor que jamais senti", respectivamente.

Como método de intervenção foi utilizado o Programa de Tratamento Stress Loading e uma cartilha com orientações, elaborada pelas autoras, sobre o programa e sobre como este poderia ser realizado no domicílio Esta técnica de tratamento, com base no Modelo Biomecânico, consiste no estresse muscular de tração e compressão ativa, e foi criado por Kirk Watson em 1987 para produzir estímulos estressantes à extremidade afetada sem movimento articular, que normalmente nestes casos são bastante dolorosos ${ }^{8}$.

O programa baseia-se em estresse e sobrecarga através do esforço muscular com máxima de resistência e por máxima permanência de tempo e carga. Esta sobrecarga na musculatura provoca uma vasodilatação reflexa sobre a vasoconstrição, característica da SCDR ${ }^{11}$. Segundo Coelho e Alves ${ }^{12: 469 ~ “(. . .) ~ o ~ e x e r c i ́ c i o ~ p r o m o v e ~ o ~ a u m e n t o ~ d e ~ i m p u l s o s ~}$ aferentes para o sistema nervoso simpático oriundo de ergorreceptores presentes no músculo quando em atividade".

O Programa de Tratamento Stress Loading fundamenta-se na manutenção do estresse da musculatura utilizando o esforço de esfregar e carregar. $O$ ato de esfregar consiste em o participante com acometimento de membro superior ficar na posição quadrupede no chão ou poderá manter-se em pé segurando uma escova de fibras grossas na mão acometida, e apoiada sobre uma mesa. O exercício é realizado aplicando o máximo de pressão, com o braço em extensão, utilizando os movimentos para frente e para trás com mínima movimentação articular durante o período de três minutos ${ }^{8}$.

Para o ato de carregar, o participante com acometimento de membro superior é orientado segurar pesos com a mão afetada, como sacolas, halteres, pacotes de alimentos, sendo realizado em pé ou sentado, em movimento ou parado, onde o indivíduo permaneça por cinco minutos carregando o peso ${ }^{11}$. A cada novo ciclo de exercícios, o tempo e a carga devem ser ajustados em contagens cada vez maiores que os ciclos anteriores.

Além do Programa de Tratamento Stress Loading os participantes receberam uma cartilha de orientações referentes à realização deste programa em domicílio (organizada pelas pesquisadoras para este estudo). Foi composta inicialmente por um espaço destinando aos dados de identificação do participante, na sequência tinham explicações sobre a realização do programa no domicílio, como uma grade de horários, onde os participantes podiam anotar o horário que executou o programa. Neste, também tinham uma EVA para o participante assinalar o nível de dor ao iniciar e ao finalizar o programa, em cada dia. A cada retorno era analisado a frequência da realizado do programa no domicílio e os níveis de dor dos participantes.

O Programa de Tratamento Stress Loading já foi utilizado como método de intervenção em dois estudos realizados por terapeutas ocupacionais. O primeiro estudo que abordou o programa foi a dissertação de mestrado da terapeuta ocupacional Miriam Delboni ${ }^{11}$, realizado com 25 participantes cometidas pela SCDR. Os resultados deste 
estudo demonstram que após o uso do programa 23 dos participantes referiram redução da dor. Outro estudo realizado por terapeutas ocupacionais que utiliza o programa como meio de intervenção é de Ponte e colaboradores ${ }^{1}$, por meio de um estudo de caso e os resultados demonstraram que o participante teve uma significativa redução da dor, o ganho de Amplitude de Movimento e a melhora do desempenho ocupacional.

\section{Descrição do Caso - Participante 1}

Participante 1 - 52 anos de idade, sexo feminino, divorciada, profissão Camareira (no momento do estudo estava afastada de suas atividades laborais), escolaridade ensino médio incompleto, com acometimento do membro superior direito (MSD), com SCDR tipo I. A síndrome foi desencadeada após realizar um procedimento de cateterismo solicitado pelo médico Angiologista, há três anos. A participante referiu dor intensa no MSD e no peito, trajeto pelo qual o cateterismo foi realizado e relata fadiga. Possui acompanhamento do ambulatório de dor, Fisioterapia e Terapia Ocupacional. A participante referiu que o quadro doloroso interfere na sua vida social e na participação ocupacional. Apresentou dificuldade para utilizar o transporte público devido a dor e condições financeiras. As atividades apontadas como as que não conseguia realizar, identificadas no DASH são: abrir um vidro com a tampa muito apertada, abrir uma porta pesada, carregar objeto pesado com mais de $5 \mathrm{~kg}$, trocar uma lâmpada acima da cabeça, lavar ou secar 0 cabelo, lavar suas costas e atividades recreativas como tricotar. Possuía os aspectos do membro com edema, cianose e alteração de temperatura.

Observou-se que na primeira avaliação o escore do DAHS do participante 1 foi de 75 na reavaliação, pós programa, o escore do participante 1 passou para 67.

O participante 1 apresentou evolução na atividade escrever, em que no início do tratamento relatava grau 3, na qual havia dificuldade média para realizar e após o tratamento passou a graduar 2, com pouca dificuldade. Colocar algo em uma prateleira acima da cabeça apresentava grau 5, em que não conseguia realizar e pós tratamento grau 4, com muita dificuldade, mas realizava. Nas tarefas domésticas no início referiu nível 4 e pós intervenção grau 3, em que realizou com dificuldade média. Arrumar a cama com grau 4, passando a graduar 2, esta é a atividade que apresentou maior ganho funcional relacionada as dificuldades. Carregar uma sacola não conseguia realizar e pós tratamento passou a executar com dificuldade. Utilizar a faca apresentava grau de dificuldade 4 e após passar pelo programa passou a ter grau 3.

Em relação a EVA na primeira avaliação o participante 1 referia dor grau 6 e pós aplicação do Programa de Tratamento Stress Loading grau passou a referir grau 5. 


\section{Descrição do Caso - Participante 2}

Participante 2 - 59 anos, sexo feminino, divorciada, profissão Doméstica (no momento do estudo estava afastada de suas atividades laborais), com acometimento de membro superior esquerdo (MSE) com SCDR tipo I. Em 2013, sofreu uma queda durante uma atividade doméstica em sua residência, fraturou o cotovelo e o antebraço esquerdo. Realizou três procedimentos cirúrgicos em decorrência da lesão. As atividades apontadas como as que não consegue realizar, segundo a avaliação do DASH foram: abrir um vidro com a tampa muito apertada, colocar algo em uma prateleira acima de sua cabeça, tarefas domésticas, trabalho de jardinagem, trocar uma lâmpada acima da cabeça, lavar ou secar o cabelo e lavar suas costas.

Observou-se que na primeira avaliação o escore do DAHS do participante 2 foi de 60,8 e na reavaliação, pós programa, o escore do participante 2 passou para 45,7.

A participante 2, também manifestou ganhos em atividades que demandavam movimentação articular de extensão e flexão. A AVD preparar refeição no início do tratamento foi graduada em pouca dificuldade e pós tratamento sem dificuldade para realizar. Colocar algo em uma prateleira acima da cabeça não conseguia fazer, com o tratamento passou a realizar com dificuldade. E a AVD higiene lavar as costas na primeira avaliação referiu não conseguir desempenhar $\mathrm{e}$ ao reavaliar relatou praticar com pouca dificuldade. Em relação a EVA na primeira avaliação o participante 2 relatou dor grau 8 e pós programa grau passou para 6.

\section{RESULTADOS E DISCUSSÕES}

As pessoas acometidas por altos níveis de dor podem apresentar comprometimentos funcionais. As atividades mais afetadas são as que exigem movimentos de extensão, flexão, rotação externa e abdução de ombro, Ponte et al. ${ }^{1}$, complementa citando os comprometimentos nos movimentos de extensão e flexão do cotovelo e preensão palmar e pinça. Estes comprometimentos podem limitar as ocupações no cotidiano no que se refere as áreas do desempenho ocupacional do autocuidado, produtividade e lazer.

As duas participantes apresentaram rigidez articular, este quadro corrobora com os dados encontrados em dois estudos realizados por Ponte et $a^{1,2}$, neste, além da rigidez articular os autores ainda ressaltam que as pessoas acometidas por esta síndrome apesentam diminuição da força, do tônus muscular, da amplitude de movimento.

O intenso quadro doloroso pode levar as pessoas acometidas pela SCDR I a ter medo de realizar movimentos (cinesiofobia) com o membro acometido, sendo este um comportamento que pode contribuir para o agravamento do quadro de rigidez articular ${ }^{13}$. Diante desta realidade, torna-se importante ressaltar que o acompanhamento de sujeitos 
acometidos por SCDR I deve ser realizado por equipes multiprofissionais, o mais precocemente possível e considerando aspectos biológicos, psicológicos/emocionais e sociais da pessoa acometida pela síndrome ${ }^{14}$.

Os escores do DASH demonstram que as duas participantes apresentam limitações e restrições para realizar as suas atividades do autocuidado, produtividade (trabalho) e lazer. Pode-se perceber na descrição dos casos que as duas participantes estavam afastadas de seus postos de trabalho devido ao quadro doloroso. Ressalta-se que o afastamento do ambiente de trabalho pode impactar a vida cotidiana das pessoas, levando a perda da identidade profissional, inseguranças relacionadas a manutenção do emprego, problemas econômicos e emocionais (sentimento de inutilidade e isolamento social). A SCDR I e os impactos causados por esta nas atividades de autocuidado, produtividade e lazer geram nas pessoas acometidas um sofrimento constante ${ }^{2}$.

Após seis semanas de intervenções terapêuticas ocupacional com a utilização do Programa de Tratamento Stress Loading, observou-se que as duas participantes apresentaram melhora no escore do DASH. Pode-se considerar pequena as alterações na funcionalidade das participantes, porém, é válido ressaltar que o programa foi aplicado durante seis semanas e ambos os participantes apresentavam quadro clínico crônico, onde a lesão havia ocorrido há anos. Mas, mesmo sobre estas condições torna-se perceptível a melhora do quadro da SCDR I utilizando o Programa de Tratamento Stress Loading como técnica de tratamento complementar.

Pode-se observar que ambas participantes apresentaram diminuição da intensidade da dor. Um estudo realizado por Delboni ${ }^{11}$ com 25 sujeitos diagnosticados com SCDR, discute a eficácia do Programa de Tratamento Stress Loading para a redução do quadro doloroso, neste estudo também se utilizou a EVA para mensurar a dor, sendo que a média inicial foi de 9,24 e na reavaliação foi de 0,18. Em seu estudo Delboni também orientou os participantes a realizar o programa no domicílio, como solicitado aos participantes deste estudo. De acordo com Jiménez ${ }^{15}$ o programa promove a redução da dor devido à inibição por via reflexa.

Ao final das intervenções com o programa, Delboni ${ }^{11}$ constatou que os participantes apresentaram melhora nos movimentos de extensão e flexão nos seguimentos de punho, cotovelo e dedos. O Programa de Tratamento Stress Loading por ser um programa baseado no estresse muscular de tração e na compressão ativa, na qual promove 0 aumento da amplitude articular, devido provocar uma vasodilatação reflexa e inibidora do ciclo vasoconstritor. Portanto, este bombeamento para drenagem linfática faz com que ocorra diminuição do edema, e diante disso a redução da dor, aumentando os movimentos articulares ${ }^{11}$. Com a melhora da função biomecânica do membro superior, as atividades cotidianas se estabelecem, ocorrendo significativa melhora nas questões psicoemocionais e socais das pessoas acometidas pela Síndrome. 
Segundo Coelho e Alves ${ }^{12}$ "o exercício promove 0 aumento de impulsos aferentes para o sistema nervoso simpático oriundo de ergorreceptores presentes no músculo quando em atividade" (p. 469). O uso da sobrecarga sensorial consiste em exercícios ativos, onde necessite o mínimo de movimentação articular e o máximo de exercício fatigante no membro envolvido.

O Programa de Tratamento Stress Loading, ainda é pouco utilizado por terapeutas ocupacionais e demais profissionais da reabilitação em suas práticas de atenção à saúde com pessoas acometidas por SCDR I. Mas este é um programa que pode se tornar uma das técnicas de intervenção quando este profissional inicia o cuidado com pessoas acometidas por esta síndrome, pois este proporciona estímulos motores que poderão contribuir para a melhora na realização dos movimentos do membro acometido, e assim, consequentemente refletirá no desempenho das atividades cotidianas da pessoa acometida pela SCDR I.

\section{CONSIDERAÇÕES FINAIS}

Observou-se que a intensidade do quadro doloroso causou comprometimento no desempenho ocupacional, com alterações de componentes motores importantes para a realização do autocuidado, da produtividade, especificamente, o trabalho e lazer das participantes deste estudo. Diante do exposto neste estudo pode-se perceber que o Programa de Tratamento Stress Loading contribuiu para a melhora da funcionalidade e minimização do quadro doloroso de ambos os participantes acometidas pela SCDR I. O Programa proporcionou uma recuperação dos componentes motores envolvidos nos casos descritos, refletindo na forma de realizarem suas atividades envolvidas no planejamento diário do autocuidado, trabalho e lazer. No entanto, este programa ainda é pouco utilizado nas intervenções terapêuticas ocupacionais. Observa-se também que este é pouco difundido nos meios acadêmicos, pois existem poucos estudos que discutem a técnica, sendo assim, sugere-se que outros estudos com essa temática sejam realizados, apontando os benefícios do programa na funcionalidade das pessoas acometidas pela SCDR I.

Este estudo tem como ponto limitantes o número pequeno de participantes, a demora destes para chegar ao cuidado terapêutico ocupacional, pois foram encaminhados tardiamente e apresentavam um quadro clínico crônico e 0 curto prazo de intervenção, que foi de seis semanas, pois acredita-se que com um período maior de acompanhamento pode ser possível obter maior eficácia e resultados positivos. Os pontos positivos do estudo foram a melhora no quadro álgico, do ganho funcional para desempenhar as atividades desejadas pelas participantes e a adesão das duas participantes a proposta do estudo. 


\section{REFERÊNCIAS}

1. Ponte AS, Duarte BSL, Godoy MCM, Delboni MCC, Costa TR. Síndrome Complexa de Dor Regional do tipo I do membro superior: tratamento baseado no Estresse Muscular de Tração e Compressão Ativa. Cad. Ter. Ocup. UFSCar. 2015;23(1):3-13.

2. Ponte AS, Machado MA, Delboni MCC, Costa TR, Duarte BSL. Complex regional pain syndrome type I: impact on work activities of working age individuals. Case reports. Rev Dor. 2017;18(3):279-85.

3. International Association for the Study of Pain. Classification of chronic pain: descriptors of chronic pain syndromes and definitions of pain terms. 2thed. Seatle: IASP Press, Washington, 1994.

4. International Association for the Study of Pain. International Association for the Study of Pain. Seattle: IASP. 2011. Disponível em: < http://www.iasp-pain.org//AM/Template.cfm?Section=Home>. Acessado em 27 de jun. 2017.

5. Patterson RW, Li Z, Smith BP, Smith TL, Koman LA. Complex regional pain syndrome of the upper extremity. Hand Surg Am. 2011;36(9):1553-62.

6. Miranda CC, Seda Junior LF, Pelloso LR. Nova classificação fisiológica das dores: o atual conceito de dor neuropática. Rev. Dor. 2016;17(Suppl 1):2-4.

7. Gaspar AT, Antunes F. Type I complex regional pain syndrome. Acta Med Port. 2011;24(6):1031-40.

8. Watson HK, Carlson L. Treatment of reflex sympathetic dystrophy of the hand with an active stress loading program, J Hand Surg. 1987;12(1):779-85.

9. Santos J, Gonçalves RS. Adaptação e validação cultural da versão portuguesa do Disabilities of the Arm Shoulder and Hand - DASH. Rev Port Ortop e Traumatol. 2006; 14(3):29-44 
10. Radomski MV. Avaliando o Contexto: Pessoal, Social e Cultural. In: Trombly, CA, Radomski M. V. Terapia Ocupacional para Disfunções Físicas. São Paulo: Santos, 2005. p.284-309.

11. Delboni MCC. Tratamento da distrofia simpático reflexa no membro superior pelo programa de reabilitação baseado no estresse muscular de tração e compressão ativa. Cadernos. Faculdades Integradas São Camilo. 2002;8(3):62-68.

12. Coelho CCS, Alves CRP. Distrofia Simpático-Reflexa. In: Freitas PP. Reabilitação da Mão. São Paulo: Atheneu, 2005. p.459-79.

13. Antunes RS, Macedo BG, Amaral TS, Gomes HA, Pereira LS, Rocha FL. Dor, cinesiofobia e qualidade de vida em pacientes com lombalgia cronica e depressao. Acta Ortop Bras. 2013;21(1):27-9.

14. Silva JA, Ribeiro-Filho NP. A dor como um problema psicofisico. Rev Dor. 2011;12(2):138-51.

15. Jiménez, MPG, Viñolo Gil MJ, Iglesias Alonsoc A, Martínez Martínez I, Aguilar Ferrándize E, Crespo Rica SA. Síntomas y tratamiento del Síndrome de dolor regional complejo. Fisioterapia, 2009;31(2):60-4. 